\title{
Blow-up points to one phase Stefan problems with Dirichlet boundary conditions
}

\section{Toyohiko Aiki}

Department of Mathematics, Faculty of Education, Gifu University, Yanagido, Gifu, 501-11, Japan. Phone: 81-58-293-2239.

Fax: 81-58-293-2243. E-mail: aiki@cc.gifu-u.ac.jp

and

\section{Hitoshi Imai}

Faculty of Engineering, University of Tokushima, 2-1 Minami-josanjima, Tokushima 770, Japan. Phone: 81-886-56-7541. Fax: 81-886-24-6799. E-mail: imai@pm.tokushima-u.ac.jp

\begin{abstract}
We study one-phase Stefan problems for semilinear parabolic equations with Dirichlet boundary conditions in one-dimensional space. We show behavior of free boundaries of blow-up solutions at finite blow-up time and numerical experiments for our problem.
\end{abstract}

Keywords

One-phase Stefan problem, blow-up solution, behavior of free boundary

\section{INTRODUCTION}

Let us consider the following one-phase Stefan problem $D P$ (resp. $N P$ ) with homogeneous Dirichlet (resp. Neumann) boundary condition in one-dimensional space: The problem is to find a curve $x=\ell(t)>0$ on $[0, T],(0<T<\infty)$, and a function $u=u(t, x)$ on $Q_{\ell}(T):=\{(t, x) ; 0<t<T, 0<x<\ell(t)\}$ satisfying that

$u_{t}=u_{x x}+u^{1+\alpha} \quad$ in $Q_{\ell}(T)$,

$u(0, x)=u_{0}(x)$ for $0 \leq x \leq \ell_{0}$,

$u(t, 0)=0 \quad$ for $0<t<T$,

(resp. $\frac{\partial}{\partial x} u(t, 0)=0$ for $0<t<T$,)

$u(t, \ell(t))=0$ for $0<t<T$,

$\frac{d}{d t} \ell(t)=-u_{x}(t, \ell(t))$ for $0<t<T$,

$\ell(0)=\ell_{0}$ 
where $\alpha$ and $\ell_{0}$ are given positive constants and $u_{0}$ is a given initial function on $\left[0, \ell_{0}\right]$.

In Fasano-Primicerio(1979) they established the local existence in time and the uniqueness for solutions to the above $D P$ and $N P$ in the classical formulation (which means that $u_{t}$ and $u_{x x}$ are continuous functions). Besides, for solutions of $D P$ and NP in the distribution sense (which means that $u_{t}$ and $u_{x x}$ belong to $L^{2}$-class) the existence, the comparison and the behavior were studied by Aiki(1990), Kenmochi(1990) and Aiki-Kenmochi(1991).

It is well known that there are blow-up solutions of the usual initial boundary value problem for semilinear equation (1) in a bounded domain (cf. Tsutsumi(1972)). Accordingly, by using comparison principle it is clear that $D P(N P)$ has a blow-up solution for a sufficiently large initial data. Here, we note the the following global existence result of a solution: Let $\left[0, T^{*}\right)$ be the maximal interval of existence of the solution to $D P$ and $N P$, we see (cf. Aiki-Kenmochi(1991)) that the following cases (a) or (b) must occur:

(a) $T^{*}=+\infty$;

(b) $\quad T^{*}<+\infty$ and $|u|_{L^{\infty}\left(Q_{\ell}(t)\right)} \rightarrow+\infty$ as $t \uparrow T^{*}$.

However, from the above result we can get no information for the behavior of free boundary $\ell$ at blow-up time. In the present paper we shall show the behavior of blow-up solutions to $D P$ and NP at finite blow-up time (see Theorems 2 and 3 ). Our proofs to Theorems 2 and 3 are essentially due to Friedman-McLeod(1985). Also, we get an estimates for the time-derivative of free boundary, $\ell^{\prime}$ (see Theorem 4).

In the final section we shall show numerical experiments to $D P$. For the investigation of the influence of a free boundary, numerical results are compared with those of the normal blow-up problem with the fixed boundary and the homogeneous Dirichlet boundary conditions.

We begin with the precise definition of a solution to $D P$ and $N P$. In this paper we consider classical solutions to our problems since we shall apply the strong maximum principle for the proofs to our theorems. Let $C^{1,0}\left(\overline{Q_{\ell}(T)}\right)$ be the set of functions which are continuous on $\overline{Q_{\ell}(T)}$ with their $x$-derivatives.

Definition 1 A couple $\{u, \ell\}$ of functions $u=u(t, x)$ and $x=\ell(t)$ is said to be a solution of $D P$ (resp. NP) on a compact interval $[0, T], 0<T<+\infty$, if the following conditions (S1) and (S2) are satisfied:

(S1) $\quad \ell \in C^{1}([0, T])$, and $u \in C^{1,0}\left(\overline{Q_{\ell}(T)}\right), u_{x x}$ and $u_{t}$ are continuous in $Q_{\ell}(T)$;

(S2) $\quad(1) \sim(3)$ and $(5) \sim(7)$ (resp. (1) (2) and (4) (7)) hold in the classical sense.

Also, we call a couple $\{u, \ell\}$ is a solution of $D P\left(\right.$ resp. NP) on an interval $\left[0, T^{\prime}\right), 0<T^{\prime} \leq$ $\infty$, if it is a solution of $D P\left(\right.$ resp. NP) on $[0, T]$ in the above sense for any $0<T<T^{\prime}$.

First, we recall the theorem concerned with local existence of solutions to the above $D P$ and $N P$.

Theorem 1 (cf. Fasano-Primicerio(1979)) We assume that $u_{0} \in C^{1}\left(\left[0, \ell_{0}\right]\right), u_{0} \geq 0$ on $\left[0, \ell_{0}\right], u_{0}\left(\ell_{0}\right)=0$ and $u_{0}(0)=0\left(\right.$ resp. $\left(u_{0, x}(0)=0\right)$. Then there exists a positive number $T_{0}$ depending only on $\left|u_{0}\right|_{C^{1}\left(\left[0, \ell_{0}\right]\right)}, \ell_{0}$ and $\alpha$ such that problem $D P($ resp. NP) has a unique solution $\{u, \ell\}$ on $\left[0, T_{0}\right]$. 
For the problems $D P$ and $N P$, we say that $[0, T), 0<T \leq+\infty$, is the maximal interval of existence of the solution, if the problem has a solution on time-interval $\left[0, T^{\prime}\right]$, for every $T^{\prime}$ with $0<T^{\prime}<T$ and the solution can not be extended in time beyond $T$.

\section{MAIN RESULTS}

In order to establish results concerned with the behavior of blow-up solutions we give assumptions $(\mathrm{H} 1) \sim(\mathrm{H} 4)$ for initial data $\ell_{0}$ and $u_{0}$.

(H1) $\ell_{0}>0$ and $u_{0} \in C^{2}\left(\left(0, \ell_{0}\right)\right) \cap C^{1}\left(\left[0, \ell_{0}\right]\right)$ and $u_{0}(x)>0 \quad$ for $x \in\left(0, \ell_{0}\right)$,

(H2) $u_{0, x x}(x)+u_{0}^{1+\alpha}(x) \geq 0$ for $x \in\left(0, \ell_{0}\right)$,

(H3) $u_{0}\left(\ell_{0}\right)=0, u_{0, x}<0$ for $x \in\left(0, \ell_{0}\right)$ and $u_{0, x}(0)=0$,

(H4) $u_{0}(0)=u_{0}\left(\ell_{0}\right)=0, u_{0, x}>0$ on $\left[0, x_{0}\right)$ and $u_{0, x}<0$ on $\left(x_{0}, \ell_{0}\right]$ for some $x_{0} \in\left(0, \ell_{0}\right)$.

Theorem 2 (cf. Aiki(to appear)) Assume that (H1) (H3) hold. Let $\{u, \ell\}$ be a solution of $N P$. If $T^{*}<\infty$, then $\ell(t) \uparrow L<+\infty$ as $t \uparrow T^{*}, u(t, 0) \rightarrow+\infty$ as $t \uparrow T^{*}$, and for any $x \in(0, L)$ there exists a positive number $M(x)$ such that

$$
|u(t, x)| \leq M(x) \quad \text { for any } t \text { with }(t, x) \in Q_{\ell}(T) .
$$

In Fujita and Chen(1988) they studied the following initial boundary value problem.

$$
\begin{aligned}
& u_{t}=u_{x x}+u^{1+\alpha} \quad \text { in }(0, T) \times(0,1), \\
& u_{x}(t, 0)=0 \quad \text { for } t \in(0, T], \\
& u(t, 1)=0 \quad \text { for } t \in(0, T], \\
& u(0, x)=u_{0}(x) \quad \text { for } x \in[0,1] .
\end{aligned}
$$

They showed that under the similar assumptions for $u_{0}$ to $(\mathrm{H} 1) \sim(\mathrm{H} 3)$ if the solution $u$ blows up then blow-up point is one and only one point $x=0$. In the proof of Theorem 2 we done with help of the idea in Fujita-Chen(1988).

In case the homogeneous Neumann boundary condition the maximum point is always the point $x=0$ for some initial data, so we get Theorem 2. However, in case Dirichlet boundary condition the maximum point may move so that we can not estimate the blowup point. Hence, we conclude the following theorem.

Theorem 3 (cf. Aiki-Imai(submitted)) Assume that (H1),(H2) and (H4) hold. Let $\left[0, T^{*}\right)$ be the maximal interval of existence of the solution $\{u, \ell\}$ to DP. If $T^{*}$ is finite, then either the following cases $(A)$ or $(B)$ always happens:

(A) $\ell(t) \rightarrow \ell_{\infty}$ as $t \uparrow T^{*}$ where $\ell_{\infty}$ is some positive number, there exists one and only one point $x^{*} \in\left(0, \ell_{\infty}\right)$ such that $u(t, x) \rightarrow+\infty$ as $t \uparrow T^{*}$ and for $x \in\left(0, \ell_{\infty}\right)$ with $x \neq x^{*}$ there is a positive constant $M_{1}(x)$ such that $|u(t, x)| \leq M_{1}(x)$ for $t$ with $(t, x) \in Q_{\ell}\left(T^{*}\right)$;

(B) $\ell(t) \rightarrow+\infty$ as $t \uparrow T^{*}$ and for any $x>0$ there is a positive number $M_{2}(x)$ satisfying that $|u(t, \xi)| \leq M_{2}(x)$ for $(t, \xi) \in Q_{\ell}\left(T^{*}\right) \cap\{\xi<x\}$.

The proof of Theorem 3 is done in the following way. We assume that (B) does not hold, that is, the following cases (A1) or (A2) is valid: 
(A1) There is a number $x_{1} \in(0, \infty)$ satisfying that for some sequence $\left\{t_{n}, \xi_{n}\right\} \subset Q_{\ell}\left(T^{*}\right)$, $t_{n} \uparrow T^{*}$ and $u\left(t_{n}, \xi_{n}\right) \rightarrow \infty$ as $n \rightarrow$;

(A2) there is a positive constant $L_{0}$ such that $\ell(t) \leq L_{0}$ for any $t \in\left[0, T^{*}\right)$.

Next, by using the similar argument to those of Friedman-McLeod(1985) we conclude that (A1) is a sufficient condition for (A). Similarly, we can prove that (A2) implies (A).

Remark 1 By the numerical experiments it seems that the case (B) in the statement of Theorem 3 does not occur, however, we can not prove it, theoretically.

Theorem 4 Under the same assumptions as in Theorem 3 we assume that the case(A) in the statement of Theorem 3 occurs. Then, there is a positive constant $C$ such that

$\left|\ell^{\prime}(t)\right| \leq C \quad$ for $t \in\left[0, T^{*}\right)$.

Proof. First, from Lemma 5.5 in Aiki-Imai(submitted) we observe that there are $x^{*}<$ $a<\ell_{\infty}$ and $t_{0} \in\left[0, T^{*}\right)$ such that $a<\ell\left(t_{0}\right)$ and

$|u(t, x)| \leq M_{1}(a):=M-1 \quad$ for $(t, x) \in \hat{Q}$

where $\hat{Q}=\left\{(t, x) \in Q_{\ell}\left(T^{*}\right) ; x>a, t>t_{0}\right\}$

Here, we denote by $v$ a solution of the following initial boundary value problem in non-cylindrical domain $\hat{Q}$;

$v_{t}-v_{x x}=M^{1+\alpha} \quad$ in $\hat{Q}$,

$v\left(t_{0}, x\right)=v_{0}(x) \quad$ for $x \in\left(a, \ell\left(t_{0}\right)\right)$,

$v(t, a)=M \quad$ for $t \in\left(t_{0}, T^{*}\right)$,

$v(t, \ell(t))=0 \quad$ for $t \in\left(t_{0}, T^{*}\right)$

where $v_{0} \in C^{1}\left(\left[a, \ell\left(t_{0}\right)\right]\right.$ with $u\left(t_{0}, \cdot\right) \leq v\left(t_{0}, \cdot\right) \leq M$ on $\left[a, \ell\left(t_{0}\right)\right], v_{0}(a)=M$ and $v_{0}\left(\ell\left(t_{0}\right)\right)=$ 0 .

By using comparison principle we see that $u \leq v$ on $\hat{Q}$ and $v_{x}(t, \ell(t)) \leq u_{x}(t, \ell(t)) \leq 0$ for $t \in\left[t_{0}, T^{*}\right)$.

Next, let $w$ be a solution to the following problem:

$w_{t}-w_{x x}=0 \quad$ in $\left(t_{0}, T^{*}\right) \times\left(a, \ell\left(t_{0}\right)\right):=\tilde{Q}$,

$w\left(t_{0}, x\right)=w_{0}(x) \quad$ for $x \in\left(a, \ell\left(t_{0}\right)\right)$,

$w(t, a)=M \quad$ for $t \in\left(t_{0}, T^{*}\right)$,

$w\left(t, \ell\left(t_{0}\right)\right)=0 \quad$ for $t \in\left(t_{0}, T^{*}\right)$

where $w_{0} \in C^{1}\left(\left[a, \ell\left(t_{0}\right)\right]\right)$ with $0 \leq w_{0} \leq v_{0}$ on $\left[a, \ell\left(t_{0}\right)\right], w_{0}(a)=M$ and $w_{0}\left(\ell\left(t_{0}\right)\right)=0$.

It is clear that $\left|w_{x}(t, x)\right| \leq C_{1}$ on $\tilde{Q}$ for some positive constant $C_{1}, w \leq v$ on $\tilde{Q}$ and $w_{x}(t, a) \leq v_{x}(t, a)$ for $t \in\left[t_{0}, T^{*}\right)$ so that

$v_{x}(t, a) \geq-C_{1} \quad$ for $t \in\left[t_{0}, T^{*}\right)$.

Putting $z(t, x)=v(t, x)+K \exp (x-\ell(t))$ where $K=\left(M^{1+\alpha}+\left|v_{x}\left(t_{0}\right)\right|_{L^{\infty}\left(a, \ell\left(t_{0}\right)\right)}+\right.$ $\left.C_{1}\right) \exp \left(\ell_{\infty}\right), z$ satisfies that $z(t, \ell(t))=K$ for $t_{0} \leq t<T^{*}$ and 
$z_{t}-z_{x x}<0 \quad$ in $\hat{Q}$,

$z_{x}\left(t_{0}, x\right)>0$ for $a \leq x \leq \ell\left(t_{0}\right)$,

$z_{x}(t, a)>$ for $t_{0}<t<T^{*}$.

Then, we conclude that $z$ takes its maximum value on any point in the curve $\{(t, x) ; x=$ $\left.\ell(t), t_{0} \leq t<T^{*}\right\}$. Hence, $z_{x}(t, \ell(t)) \leq 0$ for $t_{0} \leq t<T^{*}$. By the definition of $z$, we have $v_{x}(t, \ell(t)) \geq-K \quad$ for $t \in\left[t_{0}, T^{*}\right)$.

Therefore, we obtain that $\left|\ell^{\prime}(t)\right|=\left|u_{x}(t, \ell(t))\right| \leq K$ for $t_{0} \leq t<T^{*}$. Thus this theorem is proved.

We refer to Lemma 6.3.1 in Ladyzenskaja-Solonnikov-Ural'ceva (1968) and Lemma 4.2 in Aiki(to appear) for the proof of Theorem 4 and to Aiki-Kenmochi(1991) for comparison principle.

\section{NUMERICAL RESULTS}

We carried out numerical computations to $D P$ with $\ell_{0}=1$ and $u_{0}(x)=u_{0}^{A}(x) \equiv$ $A x^{2}(x-1)^{2}$. We note (H2) is satisfied for large $A$.

Numerical computations need some additional techniques due to treatment of the unknown boundary. Therefore, we used the fixed domain method using the following mapping function

$x(t, \xi)=\frac{\ell(t)}{2}(\xi+1), \quad 0 \leq t, \quad-1 \leq \xi \leq 1$.

The free boundary problem is transformed to the following equivalent problem in the fixed domain using the variable transform.

$$
\begin{aligned}
& u_{t}(t, \xi)=\frac{4}{\ell^{2}(t)} u_{\xi \xi}(t, \xi)-\frac{2(\xi+1)}{\ell^{2}(t)} u_{\xi}(t, 1) u_{\xi}(t, \xi)+u^{1+\alpha}(t, \xi), \quad 0<t,-1<\xi<1, \\
& u(0, \xi)=u_{0}^{A}\left(\frac{\xi+1}{2}\right), \quad-1<\xi<1, \\
& u(t,-1)=0, \quad 0 \leq t, \\
& u(t, 1)=0, \quad 0 \leq t, \\
& \ell^{\prime}(t)=-\frac{2}{\ell(t)} u_{\xi}(t, 1), \quad 0<t, \\
& \ell(0)=1 \text {. }
\end{aligned}
$$

Here we should remark this problem becomes the normal blow-up problem with the fixed boundary( (8), $u(t, 0)=0$ for $t \in(0, T],(10) \sim(11))$ by setting $\ell(t) \equiv 1$ and neglecting the 2 nd term in (13) and (17). 


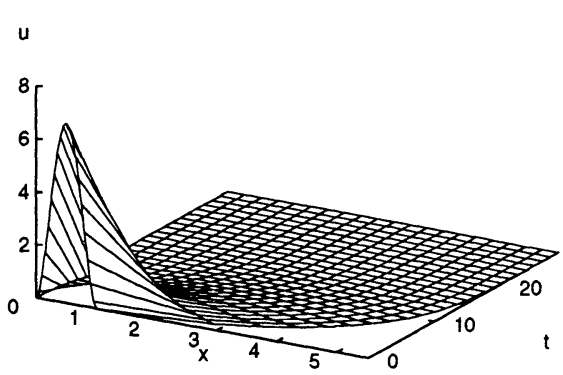

(a)

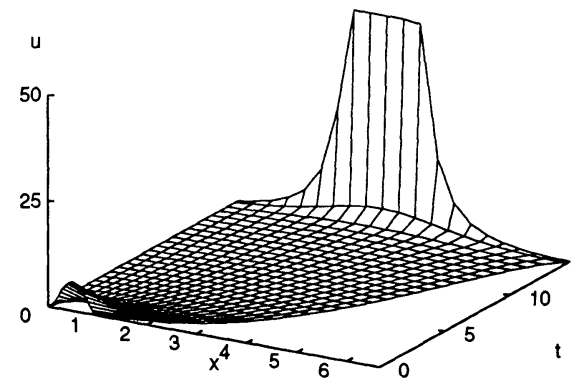

(b)

Figure $1 u(t, x)$ to $D P$ for $\alpha=1$. (a):A=108.8, $u(t, x) \downarrow 0$. (b): $\mathrm{A}=108.9, u(t, x) \uparrow+\infty$ as $t \uparrow 14.6$.

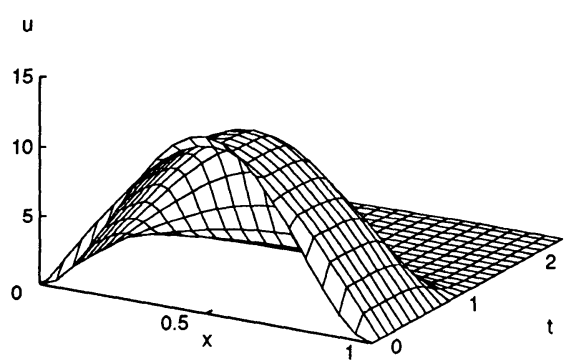

(a)

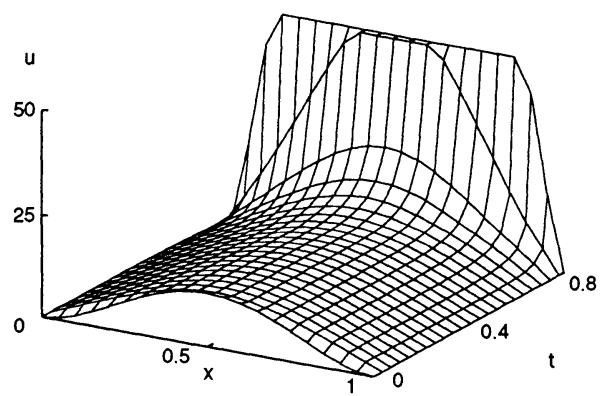

(b)

Figure $2 u(t, x)$ to the normal blow-up problem for $\alpha=1$. (a): $\mathrm{A}=205.05, u(t, x) \downarrow 0$. (b):A=205.1, $u(t, x) \uparrow+\infty$ as $t \uparrow 0.86$. 
To these transformed problems we applied the 2nd order finite difference method in space and the 4th order Runge-Kutta method in time. We used 201 grid points in space. The basic time increment is $10^{-4}$. It is controlled adaptively as in Chen (1986). Figure 1 shows numerical results to $D P$. Figure 2 shows numerical results to the normal blow-up problem with the fixed boundary $((8) \sim(10))$.

From comparison principle mentioned in the previous section it is expected the free boundary has the influence of the enhancement of the blow-up phenomena. This expectation is confirmed by these numerical results. However, the blow-up phenomena of the free boundary are not observed. This is because as Figure 1 shows blow-up points do not sufficiently get toward the free boundary.

\section{REFERENCES}

Aiki, T. (1990) The existence of solutions to two-phase Stefan problems for nonlinear parabolic equations, Control and Cybernetics, 19, 41-62.

Aiki, T. (to appear) Behavior of free boundaries blow-up solutions to one-phase Stefan problems, Nonlinear Anal. TMA.

Aiki, T. and Imai, H. (submitted) Behavior of blow-up solutions to one-phase Stefan problems with Dirichlet boundary conditions.

Aiki, T. and Kenmochi, N. (1991) Behavior of solutions to two-phase Stefan problems for nonlinear parabolic equations, Bull. Fac. Education, Chiba Univ., 39, 15-62.

Chen, Y.-G. (1986) Asymptotic behaviours of blowing-up solutions for finite difference analogue of $u_{t}=u_{x x}+u^{1+\alpha}$, J. Fac. Sci. Univ. Tokyo, Sect. IA, Math., 33, 541-574.

Fasano, A. and Primicerio, M. (1979) Free boundary problems for nonlinear parabolic equations with nonlinear free boundary conditions, J. Math. Anal. Appl., 72, 247-273.

Friedman, A. and McLeod, B. (1985) Blow-up of positive solutions of semilinear heat equations, Indiana Univ. Math. J., 34, 425-447.

Fujita, H. and Chen, Y.-G. (1988) On the set of blow-up points and asymptotic behaviours of blow-up solutions to a semilinear parabolic equation, in Analyse Mathématique et Applications, Gauthier-Villars, Paris, pp. 181-201.

Kenmochi, N. (1990) A new proof of the uniqueness of solutions to two-phase Stefan problems for nonlinear parabolic equations, Free Boundary Problems, ISNM., Vol. 95, Birkhäuser, Basel, pp. 101-126.

Ladyzenskaja, O. A., Solonnikov, V. A. and Ural'ceva, N. N. (1968), Linear and QuasiLinear Equations of Parabolic Type, Transl. Math. Monograph 23, Amer. Math. Soc., Providence R. I.

Tsutsumi, M. (1972) Existence and nonexistence of global solutions for nonlinear parabolic equations, Publ. Res. Inst. Math. Sci., 8, 211-229. 